\title{
Natural connectivity and the mitigation of cascading failures in a model of Eletrosul transmission system
}

\author{
M.S. Onetta, T. Carlotto, J.M.V. Grzybowski \\ Universidade Federal da Fronteira Sul - UFFS \\ Av. Dom João Hoffmann, 313, CEP 99.700-000, Erechim, RS. \\ E-mail: jose.grzybowski@uffs.edu.br
}

\begin{abstract}
Considering the topology of the Eletrosul transmission system, this paper investigates the impact of the addition of links upon the size and scope of cascading failures triggered by node removals. The locations of new links seek to maximize the availability of alternative closed paths within the network. The increased connectivity is shown to help mitigate the consequences of cascading failures. The results provide insightful information regarding the expansion of the electrical grid from the viewpoint of maximizing the availability of redundant closed paths within the network structure, which is believed to be related to its structural robustness.
\end{abstract}

\section{Introduction}

Electrical networks are large-scale systems whose elements feature highly interdependent behavior. Extensive and connected power-grids are more liable to enjoying the effects of inertia which, in this context, is the inclination to remain in uniformly paced global movement thus dissipating local instabilities. A central matter regarding electrical networks and network structures in general is the extent to which their associated topology can support the continuity of the processes they perform and remain operational under the failure or malfunctioning of a subset of its nodes or links. It is recognized that the robustness of network structures is closely related to the availability of redundant paths connecting nodes, such that the network can handle the redistribution of power flow after a node or line failure. Consider previous studies which found that increased number of alternatives reduces damage in the diameter a network under attack [1]. Also, the addition of redundant links was shown to improve the tolerance against coordinated attacks [2], such that robustness is argued to increase with connectivity[3][4]. Further, diverse network systems such as water distribution networks were found to have its robustness associated to the existence of redundant paths [5].

In this regard, a measure of redundant paths in a network structure is found in the concept of natural connectivity, which is regarded as a computationally affordable and physically intuitive framework to access the number and importance of closed paths between nodes [6]. The intuition behind such relation is that the greater number of alternative paths is likely to facilitate the communication between vertices as some links or nodes are damaged [6]. Due to the impact of events such as blackouts, a major concern regards how to avoid them as much as possible or, at least, how to lessen their size, scope and consequences. In the context of structural robustness, a question arises: how would increased connectivity influence the size and scope of cascading failures in the topological structure of a real power grid?

Considering the adjacency matrix corresponding to Eletrosul transmission system, this paper studies the impact of the addition of links upon the size and scope of cascading failures. The addition of new links is performed on the basis of the maximum increase in the network's natural connectivity. The size and scope of cascading failures is evaluated before and after the addition of links. The results show that, in most cases, increased connectivity helps mitigate the consequences of cascading failures. They also provide insightful information regarding the expansion of the electrical grid towards a more and more robust topological structure.

\section{Materials and methods}

As modeled according to its topology, a power grid can be represented by a graph in which nodes (generators, substations, transformers) are related to other nodes by means of links 
(transmission lines). Due to the nature of its functioning, a power grid can be modeled as a simple undirected graph $G(V, E)$ composed by nodes from a set $V$ which are linked by edges from a set $E$. The resulting graph induces a symmetrical adjacency matrix with entries $A_{i j}=A_{j i}=1$ if there is a link between nodes $i$ and $j$ and $A_{i j}=A_{j i}=0$, otherwise for $i, j=1, \ldots, N$, with $A_{i i}=0$. In such a graph, a walk of length $k$ is defined as an alternating sequence of nodes and links $v_{0} e_{1} v_{1} e_{2} \ldots e_{k} v_{k}$ with $v_{i} \in V$ and $e_{i}=\left(v_{i-1}, v_{i}\right)$; further, it is closed if the walk ends in the same link as it started, that is, $v_{k}=v_{0}$. In the calculation of the natural connectivity, the weighted sum of numbers of closed walks gives shorter closed walks increased importance by dividing the number of closed walks of a given length by the factorial of the length, as given by

$$
S=\sum_{k=0}^{+\infty} \frac{n_{k}}{k !}
$$

where $n_{k}$ the number of closed walks of length $k$ can be obtained from the $k^{\text {th }}$ power of the adjacency matrix, that is,

Equations (1) and (2) yield

$$
n_{k}=\sum_{i=1}^{N} \lambda_{i}^{k}=\operatorname{trace}\left(A^{k}\right)
$$

$$
S=\sum_{k=0}^{+\infty} \frac{n_{k}}{k !}=\sum_{k=0}^{+\infty} \sum_{i=1}^{N} \frac{\lambda_{i}^{k}}{k !}=\sum_{i=1}^{N} \sum_{k=0}^{+\infty} \frac{\lambda_{i}^{k}}{k !}=\sum_{i=1}^{N} e^{\lambda_{i}}
$$

that is referred to as Estrada index [6]. By scaling and averaging $S$, the natural connectivity of the graph is defined as

$$
\bar{\lambda}=\ln \left(\frac{1}{N} \sum_{i=1}^{N} e^{\lambda_{i}}\right)
$$

which represents an average of the eigenvalues of graph the adjacency matrix. We consider the measure in equation (4) and the adjacency matrix induced by the graph of Eletrosul transmission system [7] to evaluate the impact of the addition or removal of nodes. Towards that end, we define the variation of natural connectivity (VNC) $\Delta \bar{\lambda}$ as

$$
\Delta \bar{\lambda}=\bar{\lambda}^{C}-\bar{\lambda}
$$

where $\bar{\lambda}^{C}$ is the natural connectivity of the graph $G(V, E \cup C)$ and we take $\bar{\lambda}$ to be the natural connectivity of the unchanged graph.

Concerning cascading failures, we follow the model for the dynamics of efficiency proposed in reference [8] in which the authors considered the evolution of the efficiency of transmission over time as based on the flow redistribution after the breakdown of a node. It is assumed that the flow between any two nodes always takes the most efficient path connecting them [8]. Following such methodology, the links are modeled to reproduce the high-voltage power-grid of a real network, whose topology give rise to an undirected adjacency matrix from which the load in each node (i.e., the electrical betweenness of the node) is calculated [8]. The numerical values of the entries of the efficiency matrix belong to the interval [0,1] and, initially, the efficiency of all existing links was set as $e_{i j}=1$ as all the transmission lines are considered to be working perfectly. The efficiency of a path is modeled as the harmonic composition of the link it goes along, such that the average efficiency of the network is given by

$$
E(G)=\frac{1}{N(N-1)} \sum_{\substack{j=1 \\ j \neq i}}^{N} \frac{1}{d_{i j}}=\frac{1}{N(N-1)} \sum_{\substack{j=1 \\ j \neq i}}^{N} \varepsilon_{i j}
$$

where $d_{i j}$ is the shortest distance between nodes $i$ and $j$ and $\varepsilon_{i j}=1 / d_{i j}$ is the value of the most efficient path [8]. The load upon node $i$ at time $t$, denoted $L_{i}(t)$, is defined as the total number of most efficient paths passing through $i$ at time $t$. Further, each node is characterized by a capacity that defines the maximum load that the node can handle, given by $C_{i}=\eta L_{i}(0)$, where $\eta \geq 1$ is the tolerance parameter of the network, which is related to its capability to handle overload, and 
$L_{i}(0)$ is the initial load of node $i$. The removal of a node simulates the breakdown of an electrical substation and it changes the most efficient paths within the network, such that the efficiency matrix is changed due to the redistribution of loads over the network. This effect is modeled by the iterative rule

$$
e_{i j}(t)=\left\{\begin{array}{l}
e_{i j}(0) \frac{C_{i}}{L_{i}(t)}, \text { if } L_{i}(t)>C_{i} \\
e_{i j}(0), \quad \text { if } L_{i}(t) \leq C_{i}
\end{array}\right.
$$

such that a congestion in node $i$ at time $t$ causes the efficiency of all links passing through it to be reduced, such that energy flow will take alternative paths [8]. Finally, we assume that overloaded links and nodes are shut down, such that energy flow is reallocated and handled by remaining links and nodes. This process is iterated and the dynamics of the cascading failures indicate the route of a black-out process initiated due to the failure of a specific node. As the network reaches a steadystate, the links and nodes that remain functional correspond to the general power-grid characteristics in the period post-failure. For the simulations that follow, we considered $\eta=1.5$, which means that before the failure the system is operating at $2 / 3$ of its capacity.

\section{Results and discussion}

Figure 1 shows the values of the VNC corresponding to the addition of one edge between nodes $i$ and $j$ in the original graph of the ETS. Red-colored spots indicate links that, as created, would boost connectivity the most. Further, the original network and the altered network are shown in Figure 2. The region with new links (in green) is zoomed for clarity.

The addition of new nodes based on VNC was found to contribute to the reduction of the size and range of cascading failures for most targeted attacks, an exception being found for the node with highest degree, among few others. Figure 3 and Figure 4 show the cascading failures triggered by the removal of nodes 10 and 12, respectively, which presented the most substantial reduction in the number of degraded links (in red). The zoomed region remarks the degradation before and after the addition of links. Figure 5 shows the cascading failure triggered by the removal of node 11. The zoomed region shows the expansion of degradation as the new links are added. Note from Figure 2 that node 11 receives 6 new connections and it remains the node with highest degree after the addition of links.
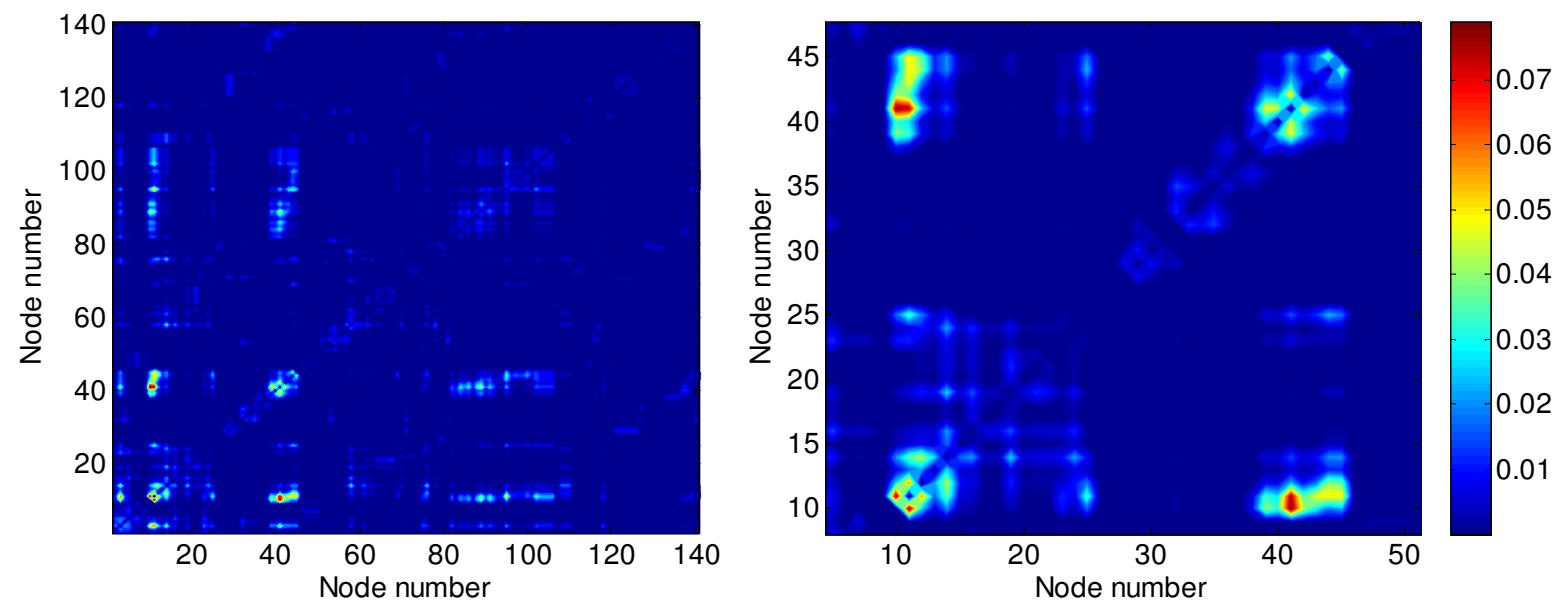

Figure 1 - Variation of natural connectivity as a function of the addition of one node: colored spots identify the position of new edges causing higher impacts upon network's natural connectivity. To the left, the contour plot for values of VNC over the set $\{1,2, \ldots, N\} \times\{1,2, \ldots, N\}$; to the right, highlight over the region with the highest values of $\mathrm{VNC}$. 


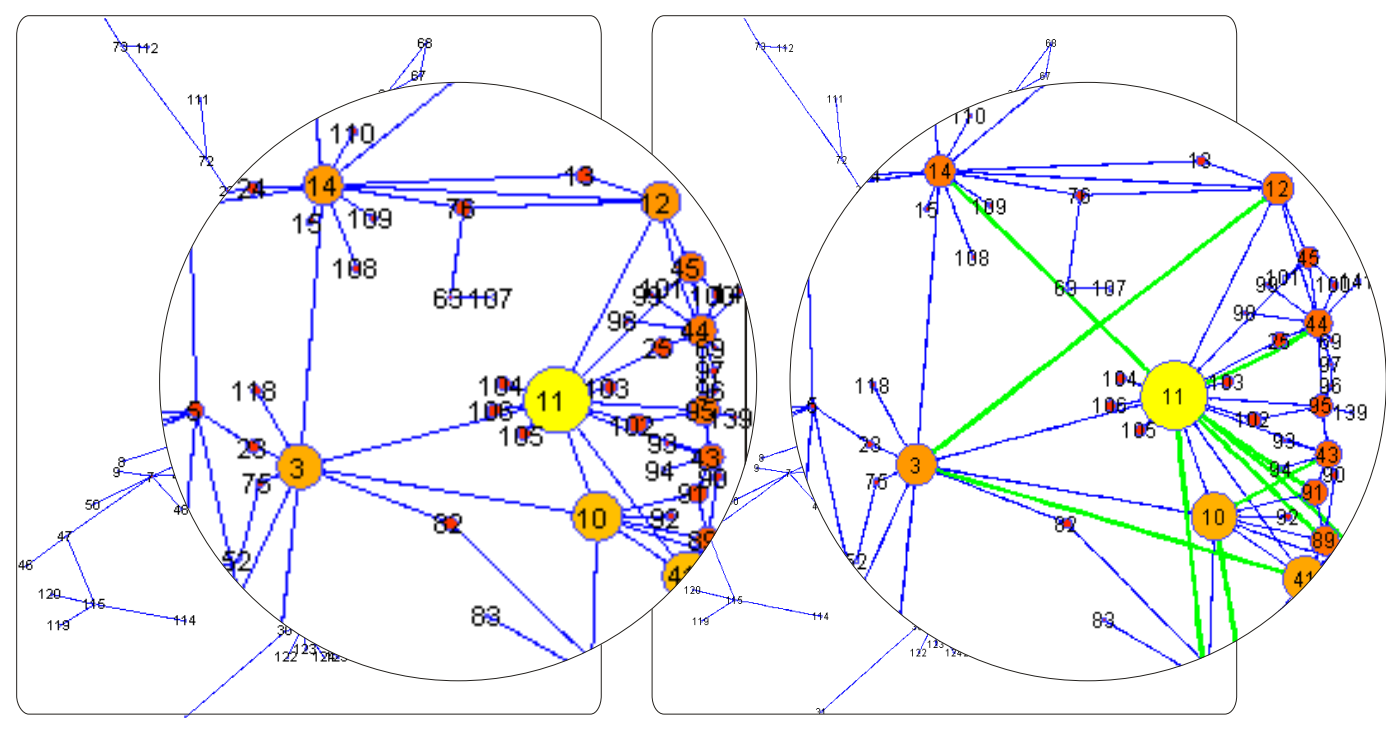

Figure 2 - Altered topology of Eletrosul transmission system after the addition of 10 new nodes that increase natural connectivity from $\cong 1.52$ to $\cong 2.22$.

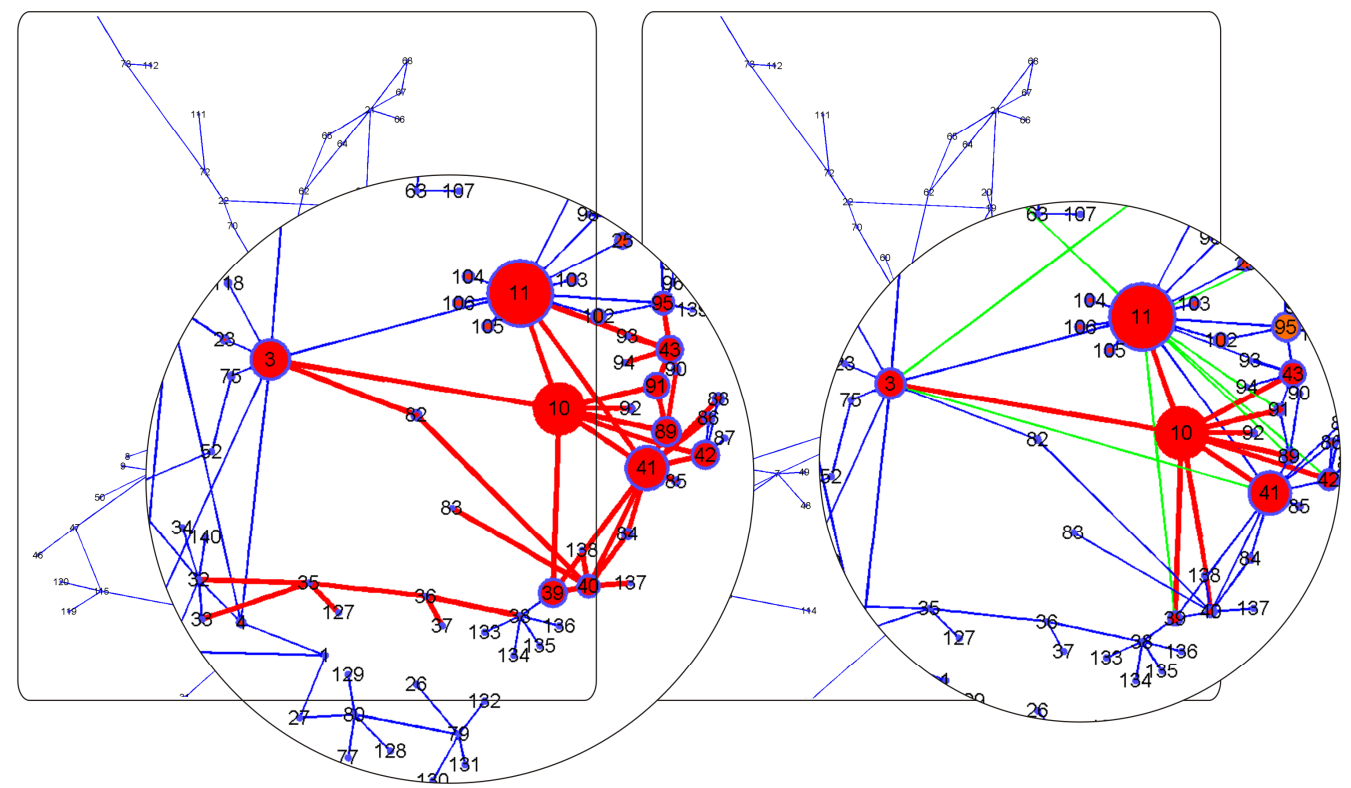

Figure 3 - Cascading failures due to the removal of node 10: the original network (to the left) experiences degradation of $21.11 \%$ of its links against $5.26 \%$ in the altered network (to the right)

From the additions based on the VNC, it can be conveyed that nodes with higher degrees have greater potential for increasing overall network connectivity. However, from the viewpoint of cascading failures, the concentration of new links upon the node with highest degree increases its load. As it eventually fails, the overload is spanned to a larger number of nodes. As some of them might not be capable of handling the load redistribution and get overloaded, they also fail and trigger a new redistribution of loads. The accumulated load can give rise to a cascading failure with greater range and lead the network to collapse. 


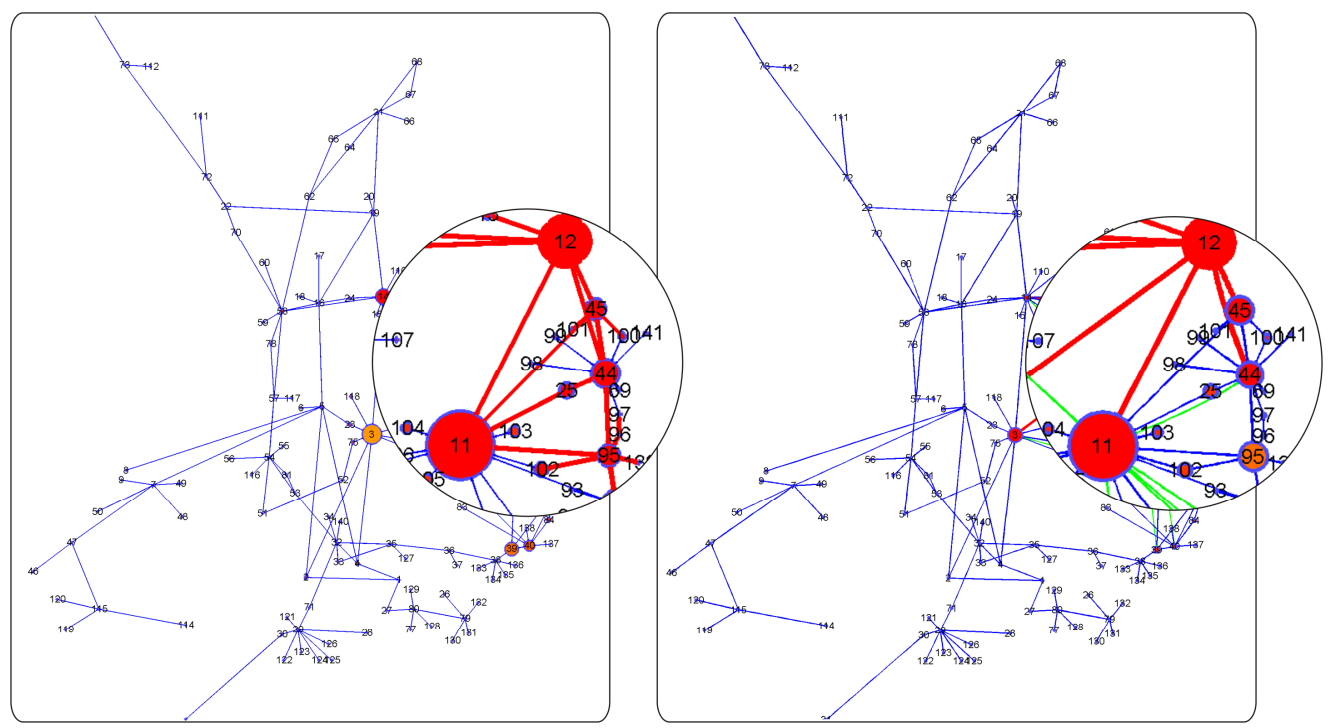

Figure 4 - Cascading failures due to the removal of node 12: the original network (to the left) experiences degradation of $10.55 \%$ of its nodes against $3.68 \%$ in the altered network (to the right).

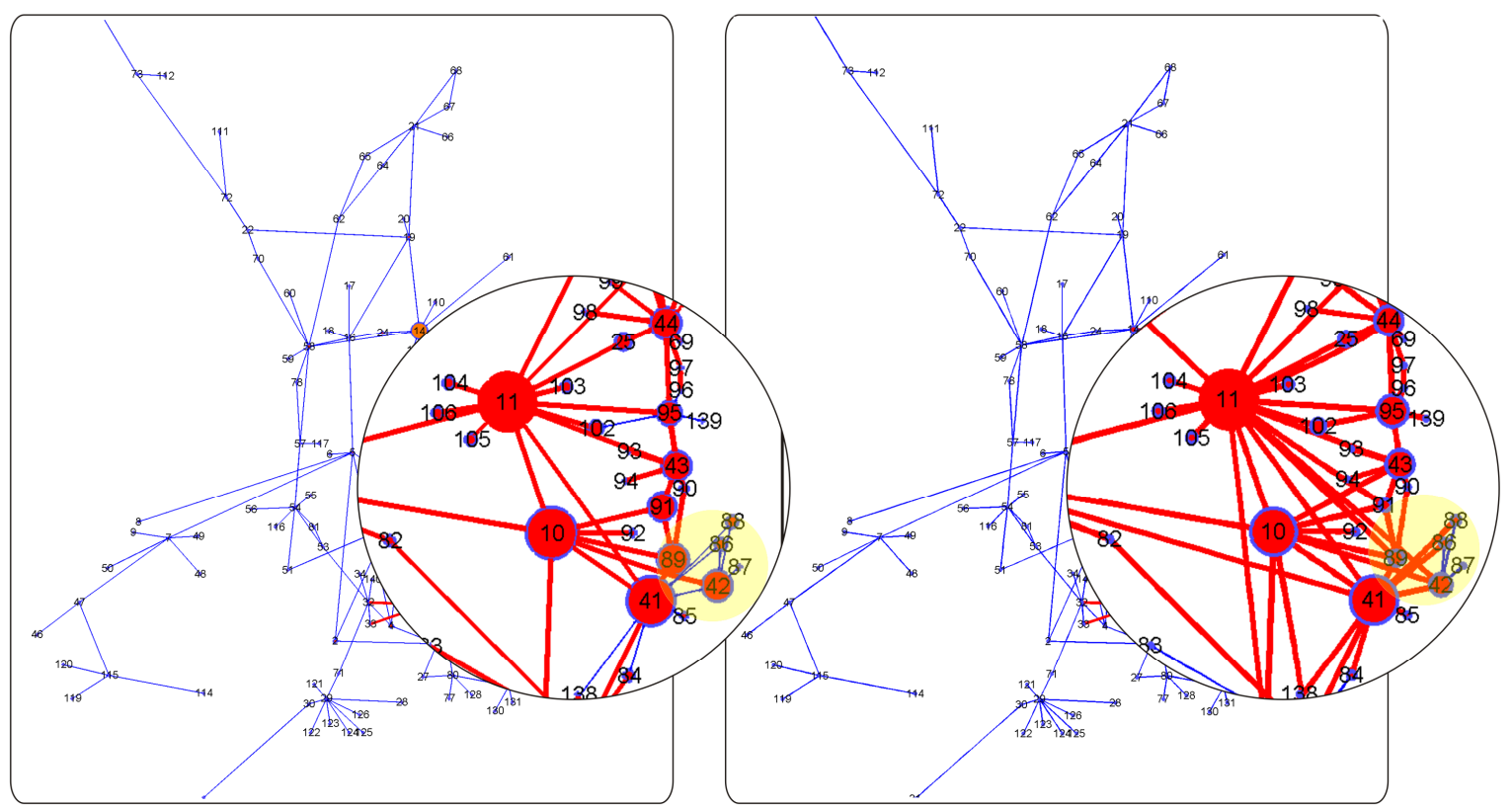

Figure 5 - Cascading failures due to the removal of node 11, the one with highest degree and subject to 6 new link additions: the failure causes increased degradation in the altered network $(37.37 \%)$ as compared to the original network $(32.22 \%)$. The highlighted spot was considerably hampered by the flow redistribution in the altered network.

Further simulations after link additions based on VNC criterion showed that the size and scope of blackouts are in general reduced relatively to those occurring for the same node removals in the original network. However, although higher connectivity and the existence of alternative paths is commonly associated to robustness, the results show that the underlying mechanics of designing a robust network is somewhat more complex than it is often assumed in the literature. In this regard, a pertinent question for investigation seems to be the relation between connectivity and criticality in the topology of power grid networks, which might hold further facts and insights related to robustness.

The authors thank FAPERGS for the financial support, grant number 12/1644-2.

Keywords: power-grids, robustness, topology, natural connectivity, Eletrosul transmission system. 


\section{References}

[1] B. Shargel, H. Sayama, I. Epstein, Y. B. Optimization of Robustness and Connectivity in Complex Networks. Physical Review Letters, volume 90, number 6, 10.1103, 2003.

[2] Y. Zhuo, Y. Peng, K. Long, Y. Liu. On allocating redundancy links to improve robustness of complex communication network, Procedigs of the SPIE 7633, Network Architectures Management and Applications VII, 76331A, doi:10.1117/12.852038, 2009.

[3] Y. Zhuo, Y. Peng, K. Long, and Y. Liu, Improving Robustness of Complex Communication Networks by Allocating Redundancy Links, in Asia Communications and Photonics Conference and Exhibition, Technical Digest (CD) (Optical Society of America), paper FD3, 2009.

[4] G. Ranjan, Z. Zhi-li, A Geometric Approach to Robustness in Complex Networks. Distributed Computing Systems Workshops, number 12137039, 2011.

[5] Yazdani, A. and Jeffrey, P. Applying Network Theory to Quantify the Redundancy and Structural Robustness of Water Distribution Systems. J. Water Resource Planning and Management, 138(2), 153-161, 2012.

[6] J. Wu, M. Barahona, Y.J. Tan, H.Z. Deng. Natural connectivity of complex networks. Chinese Physics Letters, volume 27, number 7, 078902, 2010.

[7] Map of the Electrical Transmission System, Eletrosul - Centrais Elétricas. Available online at http://www.eletrosul.gov.br/arquivos/Sistema_Transmissao_04SET2009.jpg, (2009).

[8] P. Cruciti, V. Latora, M. Marchiori, "Model for cascading failures in complex networks", Phis. Rev. E, vol. 69, 045104 (2004). 